\title{
Anabases
}

ANABASES Traditions et réceptions de l'Antiquité

$7 \mid 2008$

Varia

\section{Paul Celans Entwurf einer Landschaft und die Bedeutung der Archäologie für seine Lyrik}

\section{Matthias Steinhart}

\section{(2) OpenEdition}

1 Journals

Édition électronique

URL : http://journals.openedition.org/anabases/2473

DOI : 10.4000/anabases. 2473

ISSN : 2256-9421

Éditeur

E.R.A.S.M.E.

\section{Édition imprimée}

Date de publication : 1 mars 2008

Pagination : $91-115$

ISSN : 1774-4296

\section{Référence électronique}

Matthias Steinhart, «Paul Celans Entwurf einer Landschaft und die Bedeutung der Archäologie für seine Lyrik », Anabases [Online], 7 | 2008, Online erschienen am: 01 Dezember 2011, besucht am 10 Dezember 2020. URL : http://journals.openedition.org/anabases/2473 ; DOI : https://doi.org/10.4000/ anabases. 2473 
Anabases 7 (2008), p. 91-116.

\section{Paul Celans Entwurf einer Landschaft und die Bedeutung der Archäologie für seine Lyrik *}

MatTHias STEINHART

-, ach, die Kunst!' Ich bin, Sie sehen es, an diesem Wort Camilles hängengeblieben. Man kann, ich bin mir dessen durchaus bewußt, dieses Wort so oder so lesen, man kann verschiedene Akzente setzen:

den Akut des Heutigen, den Gravis des Historischen - auch Literarhistorischen -, den Zirkumflex - ein Dehnungszeichen - des Ewigen.

Ich setze - mir bleibt keine andere Wahl -, ich setze den Akut.“

VIER TAGE, nachdem Paul Celan am 26.1.1958 den Literaturpreis der Freien Hansestadt Bremen erhalten hatte, wurden in der Wochenzeitung Die Zeit mit einem

Für freundliche Anregungen danke ich Stefanie AREnd, Erlangen; Bernd SEIDENSTICKER, Berlin; Eckhard Wirbelauer, Strasbourg; Bernhard ZimmermanN, Freiburg. Jürgen LEHMANN, Erlangen/Freiburg, der freundlicherweise eine Manuskriptfassung gelesen hat, verdanke ich wichtige Hinweise und Verbesserungsvorschläge und Nicolai RIEDEL, Deutsches Literaturarchiv Marbach, Auskünfte zu den dortigen Bibliotheksbeständen Celans. Friedhelm PraYON, Tübingen, bin ich für die Überlassung der Vorlage für Abb. 3sehr verbunden. - Motto: P. CELAN, Der Meridian, Tübinger Ausgabe, Frankfurt/Main, 1999, p. 4. - Gedichte Celans werden nach dem leicht zugänglichen Text bei WIEDEMANN 2003 zitiert. - Abbildungsnachweise: Abb. 1: Nach S. Marinatos - M. Hirmer, Kreta, Thera und das mykenische Hellas, München, 1986, Abb. 24. Abb. 2: Nach MARINATOS Hirmer, Kreta Abb. 31. Abb. 4: Nach H. Schliemann, Mykenae, Leipzig, 1878, Abb. 327. Abb. 3: Photo F. Prayon. Abb. 5: Nach K. Vierneisel (Hrsg.), Gold der Skythen aus der Leningrader Eremitage, Ausstellungskatalog München, 1984, p. 217. - Verwendete Abkürzungen: Bollack 2000: J. Bollack, Paul Celan. Poetik der Fremdheit, Wien, 2000. - Bollack 2006: J. Bollack, Dichtung wider Dichtung. Paul Celan und die Literatur (Herausgegeben von W. WÖgERBAUER), Göttingen, 2006. - DA: Datenbank des Archivs für Antikerezeption in der deutschsprachigen Literatur nach 1945 des Seminars für Klassische Philologie der Freien Universität Berlin (http://www.antikerezeption.fuberlin.de). - Wiedemann 2003: B. Wiedemann, Paul Celan. Kommentierte Gesamtausgabe in einem Band, Frankfurt/Main, 2003. - Mit (Nr.) wird auf den Anhang verwiesen. 
Hinweis auf die Preisverleihung die Gedichte Schuttkahn sowie Entwurf einer Landschaft veröffentlicht, das 1959 in Sprachgitter nochmals vorgelegt worden ist ${ }^{1}$ :

\section{Entwurf einer Landschaft}

Rundgräber, unten. Im

Viertakt der Jahresschritt auf

den Steilstufen rings.

Laven, Basalte, weltherz-

durchglühtes Gestein.

Quelltuff,

wo uns das Licht wuchs, vor

dem Atem.

Ölgrün, meerdurchstäubt die

unbetretbare Stunde. Gegen

die Mitte zu, grau,

ein Steinsattel, drauf,

gebeult und verkohlt,

die Tierstirn mit

der strahligen Blesse.

Es ist unstrittig, dass Celan in diesem Gedicht geologische Aspekte - die schroffe, unwirtliche und vulkanische Landschaft - mit sepulkralen Elementen - einer Grabanlage - und der „Tierstirn mit / der strahligen Blesse“ zu einer Gesamtheit verbindet. Doch wird in den bisherigen Erörterungen recht kontrovers eingeschätzt, ob es sich um den völlig freien „Entwurf“ einer Landschaft handelt: So verweisen U. Baer in seiner ausführlichen Besprechung auf die Orte mehrerer Konzentrationslager oder E. Schellenberger auf das Erdinnere, in dem sie die „Rundgräber“ ansiedelt, während andere einen literari-

1 Entstanden 2.1.1958; (Nr. 45). Vgl. U. BAER, Traumadeutung, Frankfurt/Main, 2002, p. 238-256; W. IHRIG, „Landschaftsentwürfe. Paul Celan und Carl Einstein“, Archiv für das Studium der neueren Sprachen und Literaturen 136 (1984), p. 298-305; K. MANGER, "Entwurf einer Landschaft", in J. LeHMANN (Hrsg.), Kommentar zu PAUL CeLANs "Sprachgitter", Heidelberg, 2005, p. 350-357; O. PÖGGeLER, Die Frage nach der Kunst. Von Hegel zu Heidegger, Freiburg, 1984, p. 342-346; C. SAMUels, Holocaust Visions. Surrealism and Existentialism in the Poetry of Paul Celan, Columbia SC, 1993, p. 43-44; E. SCHELLENBERGER, ,Von Gletscherstuben und Meermühlen. Geologische Motive in der Lyrik Paul Celans", Wirkendes Wort 38 (1988), p. 352-353; R. TOBIAS, The Discourse of Nature in the Poetry of Paul Celan, Baltimore, 2006, p. 36-41; WiedeMANn 2003, p. 660661; zur Preisverleihung in Bremen vgl. J. Felstiner, Paul Celan. Eine Biographie, München, 1997, p. 160-161. 
schen Entwurf ohne jedes konkrete Vorbild vermuten ${ }^{2}$. Andererseits wurde aber auch mehrfach auf einen möglichen Zusammenhang mit archäologischen Stätten hingewiesen: K. Manger etwa versteht "Rundgräber" als „offensichtlich vom Menschen geschaffene Rundmonumente zur Totenbestattung (vgl. Stonehenge oder die Grabanlage auf der Burg von Mykene) 3“, und B. Wiedemann zitiert F. Behn, der in seinem von Celan benutzten Werk Kultur der Urzeit „im Zusammenhang von Stonehenge und der Grabanlage auf der Burg von Mykenae von einem ,Gräberrund“" spreche, was Celan zum Begriff der „Rundgräber" angeregt habe ${ }^{4}$; welcher Ort Aspekte des Gedichts nun allerdings tatsächlich geprägt hat, wurde doch stets offengelassen.

Die Behandlung dieser Frage zielt natürlich nicht auf ein Verständnis des Gedichts als topographischer Beschreibung, sondern auf die Benennung eines Elements, das zur Genese der eigenständigen poetischen Vorstellungswelt von Entwurf einer Landschaft beigetragen hat und zugleich in ihr aufgegangen ist. Gilt doch auch für dieses Gedicht, was Marie Luise Kaschnitz in ihrer Rede zur Verleihung des GeorgBüchner-Preises an Celan so prägnant formuliert hat: „Immer und durch alle Stilwandlungen hindurch beschwört er die innere, nicht die äußere Welt. Aber diesen inneren Erlebnissen leiht er Gestalt und Bewegung der äußeren Dinge, das ist das Ungewohnte und Faszinierende schon von Anfang an. Er hat die Außenwelt in sich hineingenommen und springt dort in der kühnsten Weise mit ihr um 5." Gleichwohl dürfte es auch in diesem Fall legitim sein, der Frage nachzugehen, womit Celan in „der kühnsten Weise“ umspringt ${ }^{6}$. Wie sich dabei zeigen wird, kann durch die Festlegung

Vgl. BAER, Traumadeutung [Anm. 1], p. 241-248; SCHELLENBERGER, „Gletscherstuben“ [Anm. 1], p. 352-353. - Völlig freie Erfindung: IHRIG, „Landschaftsentwürfe“ [Anm. 1], p. 300; ToBias, Nature [Anm. 1], p. 41; K. VosWInCKel, Paul Celan: verweigerte Poetisierung der Welt. Versuch einer Deutung, Heidelberg, 1974, p. 67.

4 WIEDEMANN 2003, p. 660 mit Nachweis.

5 M. L. KASCHNITZ, „Rede zur Verleihung des Georg-Büchner-Preises an Paul Celan am 22.10.1960", in M. L. KASCHNITZ, Zwischen Immer und Nie. Gestalten und Themen der Dichtung, Frankfurt/Main, 1971, p. 304-313; Zitat: p. 309. M. L. Kaschnitz, seit 1925 mit dem Klassischen Archäologen Guido von Kaschnitz-Weinberg verheiratet (vgl. E. Homann-Wedeking, „Guido Kaschnitz-Weinberg“, Paideuma 7 [1959], p. 11-18; R. Lullies, „Guido von Kaschnitz-Weinberg“, in R. Lullies - W. SCHIERING [Hrsg.], Archäologenbildnisse, Mainz, 1988, p. 248-249) und auch selbst an der Antike interessiert (vgl. M.L. KASCHNITZ, Griechische Mythen, Hamburg, 1943), ging auf die Bedeutung der Antike im Werk Celans indessen nicht ein. - Zur Problematik vgl. auch B. AlLEMANN, „Das Gedicht und seine Wirklichkeit“, Études germaniques 25 (1970), p. 266-274.

6 Zu diesem Ansatz BollaCK 2000, p. 222-228; BollaCK 2006, p. 18-19; 69; J.K. LYON, "'Ganz und gar nicht hermetisch': Überlegungen zum 'richtigen' Lesen von Paul Celans Lyrik“, in J. P. Strelka (Hrsg.), Psalm und Hawdalah. Zum Werk Paul Celans. Akten des Internationalen Paul Celan-Kolloquiums New York 1985, Jahrbuch für Internationale Germanistik, Reihe A, Band 20, Bern/Frankfurt am Main/New York/Paris, 1987, p. 171191, bes. p. 173-174. 
des Ortes, der verschiedenen Partien des Gedichtes zugrundeliegt, und seinen kulturellen Kontext das bisherige inhaltliche Verständnis von Entwurf einer Landschaft unterstützt werden.

Dabei weist nun eine ganze Reihe von Bestandteilen des Gedichts auf Bezüge zu Mykene, dessen Grabanlagen, den geographischen Raum und die landschaftliche Prägung sowie auf einen der spektakulären Funde Heinrich Schliemanns. Aufschlussreich sind zunächst die von Celan genannten „Rundgräber, unten“" sowie die räumliche Anlage des Gedichts mit einer ansteigenden Landschaft 7, deren Charakter den Gedanken an das in einer Ebene liegende Stonehenge wenig überzeugend erscheinen lässt. Wie bereits erwähnt, wurde „Rundgräber, unten “ aber

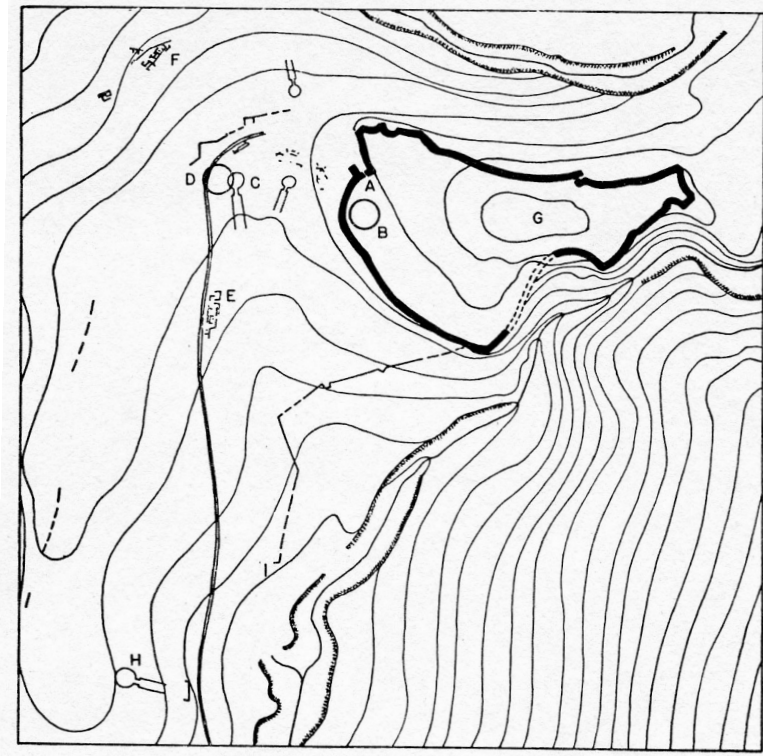

Abb. 1. Grundriss von Mykene auch schon mit Mykene und dem Gräberrund A des 16. Jhs. v. Chr. verbunden, das in den unteren Bereich der später entstandenen Burganlage einbezogen wurde (Abb. 1 : B) ${ }^{8}$. Verfolgt man den Gedanken einer Anregung durch Mykene weiter, so sind als Ausgangspunkt des Gedichts jedoch andere Gräber stimmiger: Dies sind die Tholosgräber, die sogenannten Schatzhäuser des 14. Jhs. v. Chr. mit ihren zum Teil gewaltigen Kuppelkonstruktionen (Abb. 1 : C.2) ${ }^{9}$. Die Tholosgräber liegen unterhalb des Burgbergs, was sich sowohl zu „unten“ als auch zu der ansteigend vorgestellten Landschaft besser fügt. In diesem Zusammenhang ist es nun aufschlussreich, wie Celan den Begriff „Rundgrab“ in dem Gedicht Mittags verwendet ${ }^{10}$ :

7

8 Zum Gräberrund A und den dort gemachten Funden vgl. H. SCHLIEMANN, Mykenae, Leipzig, 1878, p. 175-380 sowie M. Andronikos, Totenkult, Archaeologica Homerica. Die Denkmäler und das frühgriechische Epos Band III, Kapitel W, Göttingen, 1968, p. 77; G. KARO, Die Schachtgräber von Mykenai, München, 1930/31; S. MARINATOS M. Hirmer, Kreta, Thera und das mykenische Hellas, München, 1986, ab Taf. 184 passim. SChliemann, Mykenae [Anm. 8], p. 45-57. Vgl. Andronikos, Totenkult [Anm. 8], p. 7779; G. Gruben, Griechische Tempel und Heiligtümer, München5, 2001, p. 20-23 m. Lit. Mittags. Entstanden 30.4.1964, publiziert 1967 in Atemwende, (Nr. 41). Zum Aufenthalt Celans in Cerveteri vgl. u. 104 Ann. 56. 


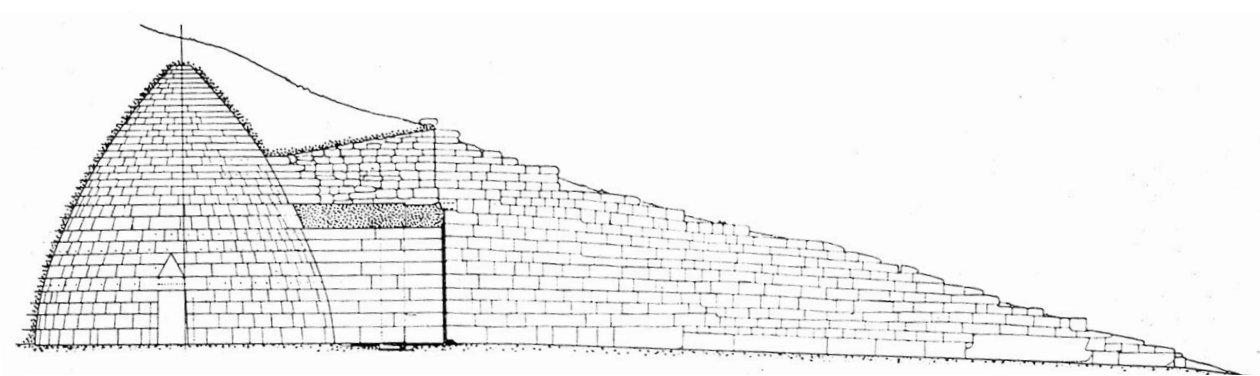

Abb. 2. Kuppelgrab („Schatzhaus des Atreus“) in Mykene.

MitTags, bei

Sekundengeflirr, im Rundgräberschatten, in meinen gekammerten Schmerz - mit dir, Herbeigeschwiegene, lebt ich zwei Tage in Rom von Ocker und Rot kommst du, ich liege schon da, hell durch die Türen geglitten, waagrecht -:

es werden die Arme sichtbar, die dich umschlingen, nur sie. Soviel Geheimnis bot ich noch auf, trotz allem.

Mit „Rundgräberschatten“ und dem „gekammerten Schmerz“ wird auf die Grabanlagen der sogenannten Banditacchia-Nekropole verwiesen, vor allem auf die großen Tumuli des 7. Jhs. v. Chr. (Abb. 3) ${ }^{11}$. Celan verwendet also „Rundgräber“ nachweislich für eine Grabform, die den mykenischen Kuppelgräbern in ihren Grundzügen ähnelt ${ }^{12}$. Da es angemessen sein dürfte, gerade bei diesem Autor von einer sehr bewussten Verwendung des Wortes auszugehen, werden mit „Rundgräber, unten“ also die Tholosgräber unterhalb der Burg von Mykene gemeint sein 13; bei „Rundgräber“ kann es sich dabei um eine der bekannten Neuschöpfungen Celans aus zwei Wörtern handeln,

11 Vgl. jetzt S. HAYNES, Kulturgeschichte der Etrusker, Mainz, 2005, p. 93-96; 173-174; F. PraYON, Die Etrusker. Jenseitsvorstellungen und Ahnenkult, Mainz, 2006, p. 11-27. Vgl. auch (Nr. 71).

12 Im übrigen war die für die Gräber des Gräberrundes A zutreffende Bezeichnung „Schachtgräber" Celan bekannt, der sie auch verwendet hat (Nr. 57); vgl. WiedEMANN 2003, p. 767.

13 Zum Sprachsystem Celans vgl. etwa BollaCK 2000, p. 81-82; 125. G. Baumann geht mehrfach auf den diffizilen Umgang Celans mit Sprache ein; vgl. G. BAUMANN, Erinnerungen an Paul Celan, Frankfurt/Main, 1992, p. 34; 97-103. 


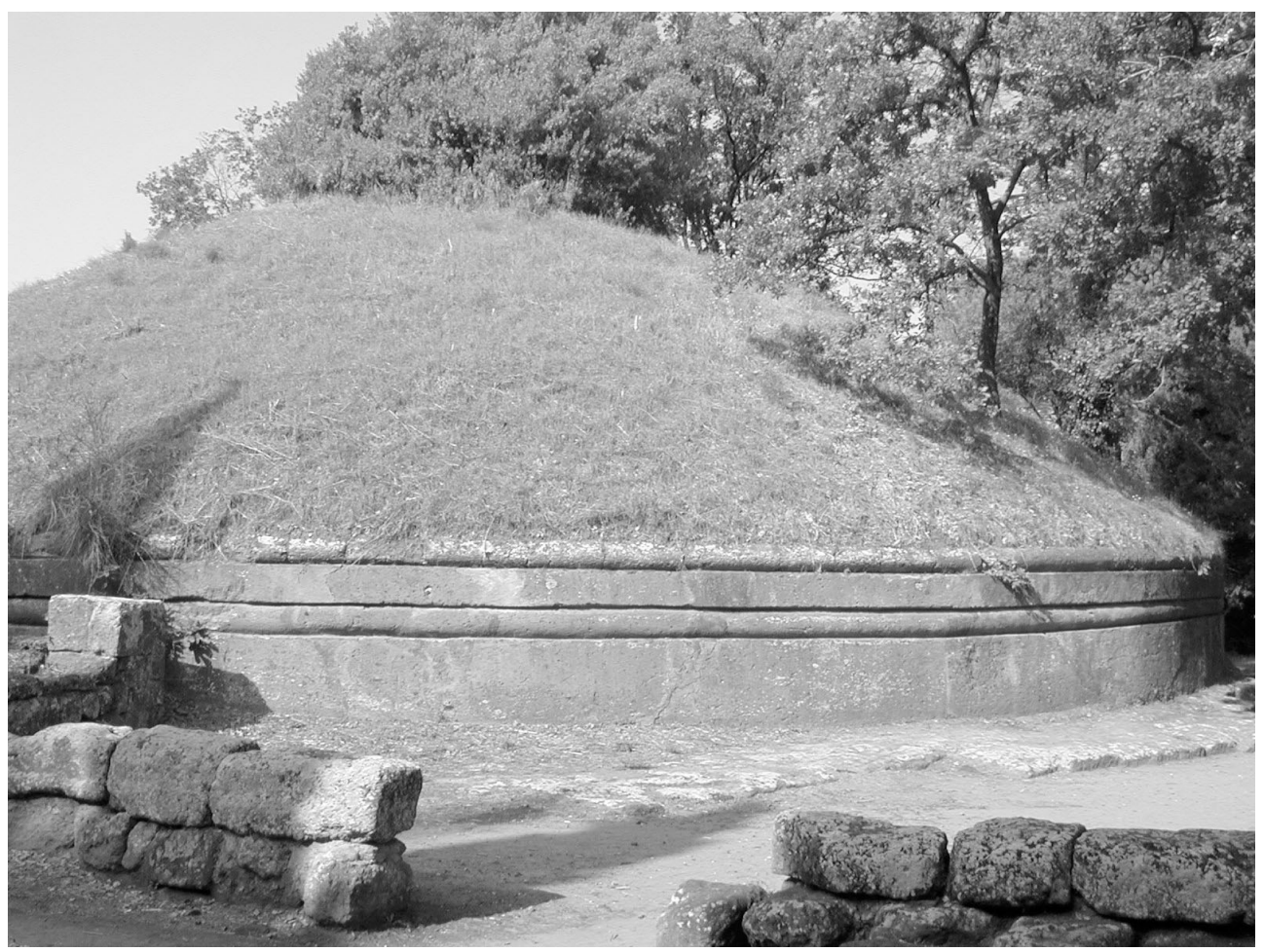

Abb. 3. Tumulus in der Nekropole von Cerveteri

die in diesem Fall aus der Behandlung der Kuppelgräber bei F. Behn abgeleitet wäre: „Die Kuppelgräber... sind idealisierte Nachbildungen des... Rundhauses 14“.

Doch damit zu weiteren Elementen von Entwurf einer Landschaft. Auf „Rundgräber, unten“ folgt „Im / Viertakt der Jahresschritt auf / den Steilstufen rings“, worauf von „Gegen / die Mitte zu, grau, / ein Steinsattel“ die Rede ist. Dies entspricht den räumlichen Gegebenheiten von Mykene, wie sie etwa Heinrich Schliemann beschrieb: „Die Lage von Mykene ist ausgezeichnet beschrieben von Homer (Odyssee, III, 263) durch $\mu \nu \chi \omega$ "А $\rho \gamma \in O 5$ iтாоßóToto im äussersten Winkel des rossenährenden Argos", denn es liegt in der Nordecke der Ebene von Argos, in einer Nische zwischen den vorerwähnten beiden erhabenen Kuppen des Berges Euboea, von wo es den obern Theil der grossen Ebene und den wichtigen Engpass beherrschte, durch welchen die Strasse nach Phlius, Kleonae und Korinth führte. Die Akropolis lag auf einer mächtigen Felshöhe ${ }^{15}$..." Um die Tholosgräber erhebt sich also auf allen Seiten

14 F. BEHN, Kultur der Urzeit Band 2. Die älteren Metallkulturen, Berlin, 1950, p. 66 [Kursivsetzung Verf.]. - Zu entsprechenden Wortverbindungen vgl. nur (Nr. 38): "Sipheten und Probyllen“.

15 Schliemann, Mykenae [Anm. 8], p. 31-32. - Vgl. auch G.E. Mrlonas, Mycenae. The capital City of Agamemnon, Princeton, 1957, p. 14-23. 
eine bergige Landschaft, wobei die Palastanlage sich auf einem Bergsattel über sechs Terrassen befindet 16. Man kann damit festhalten, dass die Vorstellung eines „Steinsattels“ oberhalb von „Rundgräbern“ mit Mykene völlig übereinstimmt.

Weniger für nur einen mediterranen Ort spezifisch sind weitere Teile des Gedichts, die sich gleichwohl mit Mykene als Anregung sehr gut verstehen lassen: So „meerdurchstäubt“, das auf das Meer verweist, das wie überall in Griechenland auch von Mykene nicht weit entfernt ist ${ }^{17}$; zu „wo uns das Licht wuchs“ kann an die verbreitete Schilderung des griechischen Lichts erinnert werden, wie sie sich unter anderem bei Hölderlin und Hugo von Hofmannsthal findet, die Celan bekanntlich beide sehr geschätzt hat ${ }^{18}$. In einer Formulierung Hofmannsthals über die griechische Landschaft sind denn auch Ähnlichkeiten zu Celans Entwurf einer Landschaft nicht zu übersehen: „Der erste Eindruck dieser Landschaft, von wo man sie betrete, ist ein strenger. Sie lehnt alle Träumereien $a b$, auch die historischen. Sie ist trocken, karg, ausdrucksvoll und befremdend wie ein furchtbar abgemagertes Gesicht: aber darüber ist ein Licht, dessengleichen das Auge nie zuvor erblickt hat ${ }^{19}$..."

Der Bezug auf Mykene lässt sich aber auch an den vorkommenden Farbwerten festmachen: „Ölgrün“ wurde bereits allgemein mit Ölbäumen verbunden, wie sie auch den Burghügel von Mykene umgeben ${ }^{20}$; die Formulierung lässt an „Ölgelände“ für einen Ölbaumhain in Rainer Maria Rilkes Gedicht Der Ölbaum-Garten denken, das Celan bereits in seiner Jugend zitiert hat; dass Celan Begriffe Rilkes in Form einer schöpferischen Neugestaltung aufgegriffen hat, ist vielfach belegt ${ }^{21}$. Und auch die

16 Vgl. E. French, Mycenae. Agamemnon's Capital, Stroud, 2002, 13-17; MARINATOS Hirmer, Kreta [Anm. 8], p. 160; Mylonas, Mycenae [Anm. 15], p. 21.

17 Vgl. VoswincKel, Celan [Anm. 2], p. 71.

18 Vgl. z. B. Friedrich HöLDERLIN, Griechenland, V. 19-21 („Aber silbern / An reinen Tagen / Ist das Licht“); zitiert nach J. SCHMIDT (Hrsg.), Friedrich Hölderlin. Sämtliche Gedichte, Frankfurt/Main, 2005, p. 421. - Hugo VON HofMANNSTHAL, Augenblicke in Griechenland, zitiert nach B. SCHOELLER in Beratung mit R. HiRSCH (Hrsg.), Hugo von Hofmannsthal, Gesammelte Werke, Band 7. Erzählungen. Erfundene Gespräche und Briefe. Reisen, Frankfurt/Main, 1979, p. 630-631.

19 Hofmannsthal, Griechenland, zitiert nach ScHOeller, Hofmannsthal [Anm. 18], p. 630.

20 Vgl. IHRIG, „Landschaftsentwürfe“ [Anm. 1], p. 303 m. Lit.; VoswinCKEL, Celan [Anm. 2], p. 71.

21 Der Ölbaum-Garten (Paris, Mai/Juni 1906). E. ZINN (Hrsg.), Rainer Maria Rilke. Sämtliche Werke. Herausgegeben vom Rilke-Archiv in Verbindung mit Ruth Sieber-Rilke. Erster Band. Gedichte, Erster Teil, Frankfurt/Main, 1955, p. 492-493; von Celan zitiert: Felstiner, Celan [Anm. 1], p. 32. - Zum Einfluss von Rilkes Werk auf Celan, der sich häufig in Anklängen und Umformungen äußert, vgl. BOLLACK 2006, p. 219-241, bes. p. 223-224; 227-228; U. FÜllebORN, „Rilke und Celan“, in I. H. SOlBrig - J. W. STORCK (Hrsg.), Rilke Heute. Beziehungen und Wirkungen, Frankfurt/Main, 1975, p. $49-70$. 
Bezeichnung „grau“ ist - wenngleich ebenfalls sehr allgemein - für den "Steinsattel“ von Mykene jedenfalls überaus passend 22 .

Schließlich kann mit Mykene als Anregung für Celan aber auch noch ein weiteres Motiv von Entwurf einer Landschaft eine primäre Erklärung finden. Das Gedicht endet mit „ein Steinsattel, drauf, / gebeult und verkohlt, / die Tierstirn mit / der strahligen Blesse". Diese Verse wurden bislang vor allem in zwei Richtungen interpretiert: K. Manger verband sie mit dem zuvor genannten, ganz wörtlich genommenen "Steinsattel“ und deutete sie als den offenbar geschundenen Pegasos 23. Weiter schien "Stirn“ als geologischer Begriff zwar dem "Sattel“ zu entsprechen, zugleich aber mit dem gleichlautenden Wort "Stern" und damit dem ,Judenstern" in Verbindung gebracht werden zu können; das Wort „Blesse“ deute danach auf Blessur 24. Wie mir scheint, liegt eine andere Anregung für die "Tierstirn mit / der strahligen Blesse“

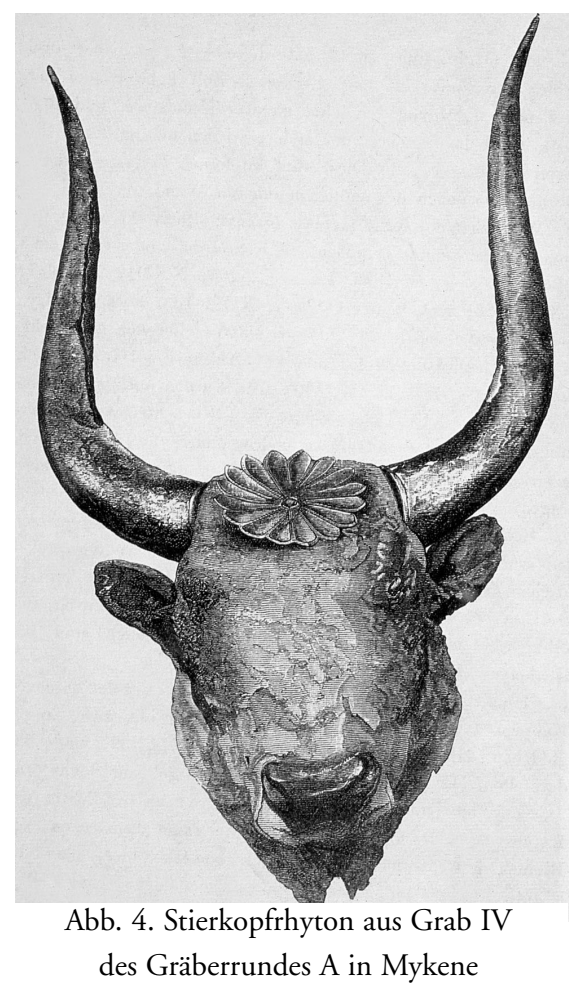
jedoch näher, die nach meinem Verständnis durch "Steinsattel, drauf“ mit dem Burgberg von Mykene verbunden ist: Dort, in Grab IV des Gräberrunds A, wurde von Heinrich Schliemann ein (ohne Hörner) 15,5 cm hohes Stierkopfgefäß aus Silber und Kupfer gefunden, das ursprünglich als Rhyton, also als Spendegefäß Verwendung fand (Abb. 4) ${ }^{25}$. Der Kopf ist als Fragment erhalten, dessen untere Halspartie wie abgerissen wirkt, und war bei seiner Auffindung verbogen (,gebeult"). Aus der schwarzen, oxydierten Oberfläche der silbernen Partien des Kopfes („verkohlt“) leuchtet neben

Vgl. etwa W. Hausenstein, Das Land der Griechen. Fahrten in Hellas, Frankfurt/Main 1934, p. 128-129: „Die Burg wird von dem Gebirge, das dahinter liegt, zuerst nicht unterschieden. Grau steht in Grau. Erst allmählich löst sich das Relief des schweren Gemäuers ab, vortretend vom Gebirge, das in zwei steilen Schrägen, mit zwei rauhen Gipfeln dahinter steht, fahlgrau im Gestein, auch beunruhigend rötlich und mit einem trauernden Violett; nur in der Tiefe grün und gelb bewachsen, in der Höhe karstig, voll von Geröll, mit gratigem Fels bekrönt." m. Abb. 327-328 sowie jetzt R. B. KoeHL, Aegean Bronze Age Rhyta, Philadelphia, 2006, 115 Nr. 294. 
anderen Partien vor allem die goldene Rosette hervor, die auf der Stirn aufgesetzt ist die „Tierstirn mit / der strahligen Blesse 26“.

In Entwurf einer Landschaft fügen sich demnach mit „Rundgräber, unten“, „Steilstufen“, „Steinsattel“, „drauf, / gebeult und verkohlt, / die Tierstirn mit / der strahligen Blesse“, mit „Licht“, „meerdurchstäubt“, aber auch mit „Ölgrün“ und „grau“ zu viele Elemente des Gedichts zu der angenommenen Anregung durch Mykene, als dass dies bloßer Zufall sein könnte ${ }^{27}$. Dass es sich um eine Anregung durch diesen Ort und um die Verwendung von atmosphärisch passenden Versatzstücken handelt und nicht etwa um eine - bei Celan so ohnehin kaum zu erwartende - ,Beschreibung' eines Ortes, ergibt sich deutlich aus den bislang ausgelassenen Versen „Laven, / Basalte, weltherz- / durchglühtes Gestein. / Quelltuff" mit ihrer so eindrucksvollen vulkanischen Unwirtlichkeit. Diese vulkanische Prägung entspricht zwar durchaus den allgemeinen geologischen Gegebenheiten Griechenlands, jedoch nicht denjenigen des Burgbergs von Mykene ${ }^{28}$. Auch hier sind - wie in vielen Gedichten Celans - unterschiedliche Elemente zu etwas Neuem gestaltet: um bei geologischen Aspekten von Celans Lyrik zu bleiben, so sei nur die Formulierung „Meermühle geht, / eishell und ungehört, / in unsern Augen“ in Weiss und Leicht genannt, der die Besprechung einer geologischen Eigenheit der griechischen Stadt Argostoli auf Kephallenia zugrundeliegt, ohne dass das Gedicht ansonsten etwas mit der Insel im Ionischen Meer oder auch nur mit Griechenland zu tun hätte 29.

26 Ich verstehe „strahlig“ damit im herkömmlichen Sinn, also wie z. B. in zehnstrahlig; vgl. auch VoswinCKel, Celan [Anm. 2], p. 71. - Da nicht nachzuweisen ist, ob Celan Schliemanns Beschreibung des Stierkopfs kannte, weise ich nur unter Vorbehalt darauf hin, dass dieser die Rosette als Sonne bezeichnet hat (SCHLIEMAnn, Mykenae [Anm. 8], p. 249): „Unmittelbar neben dem kupfernen Gefäß mit den goldenen Knöpfen fand ich einen silbernen Kuhkopf mit zwei langen goldenen Hörnern, wie es die Bilder Nr. 327 und 328 darstellen. Er hat eine schön verzierte goldene Sonne von 2 1/5 Zoll im Durchmesser auf der Stirn; in der Mitte des Kopfes ist ein rundes Loch ..."

27 Ich bin mir des möglichen Einwands bewusst, dass es für meinen Vorschlag keinen direkten Beleg gibt, doch gilt dies auch für andere Anspielungen Celans, die dieser mitunter sehr gezielt verschleiert hat; vgl. Baumann, Celan [Anm. 13], p. 31; SCHELlEnBerger, "Gletscherstuben" [Anm. 1], p. 352. - Zur Zufälligkeit der Belege in diesem Zusammenhang vgl. auch LYON, „Lesen“ [Anm. 6], p. 176; U. WERnER, Textgräber. Paul Celans geologische Lyrik, München, 1998 verweist p. 54-55 Anm. 5 auf geologische Begriffe, die in den Beständen der Bibliothek Celans nicht nachzuweisen sind.

28 Vgl. M.D. Higgins - R. Higgins, A Geological Companion to Greece and the Aegean, London, 1996, p. 46-47.

29 Weiss und leicht. Entstanden 23.10.1956 - 8.10.1957. WiEDEMANn 2003, p. 99; vgl. p. 651-652; hier zitiert V. 30-32. Zur „Meermühle“ - einer Meeresströmung, die dazu genutzt wurde, zwei Mühlen anzutreiben und seit dem frühen 19. Jahrhundert von Reisenden beschrieben wurde - vgl. SCHELlEnBERGER, „Gletscherstuben“ [Anm. 1], p. 350-351; M. Steinhart - E. Wirbelauer, Aus der Heimat des Odysseus. Reisende, Grabungen und Funde auf Ithaka und Kephallenia bis zum ausgehenden 19. Jahrhundert, Mainz, 2002, p. 199. 
Der hier vertretene Zusammenhang mit Mykene unterstützt dabei nur die bereits vollzogene Einordnung der Landschaft, deren rauher Charakter mehrfach betont worden ist und die mit „Laven, Basalte, weltherz- / durchglühtes Gestein“ wie mit „gebeult und verkohlt, / die Tierstirn" auf gewaltsame Vorgänge verweist, die „Rundgräber“ und vielleicht auch „die / unbetretbare Stunde“ zudem auf Tod und Vergänglichkeit ${ }^{30}$. Die Verbindung mit Mykene lässt den Charakter des Gedichts noch deutlicher hervortreten: Mykene als Sitz des Geschlechts des Atreus ist wie kein anderer Ort des griechischen Mythos mit Blutschuld, Inzest und Mord verbunden, oder mit Zbiegniew Herbert „die griechische Hauptstadt des Verbrechens. Selbst dem, dessen Gepäck an mythologischen Reminiszenzen bescheiden ist - macht Mykene unabweislich den Eindruck eines von der Tragödie heimgesuchten Ortes ${ }^{31}$...". Celan hat diese Tradition genutzt, um seinen recht düsteren Entwurf einer Landschaft anzulegen ${ }^{32}$. Berücksichtigt man nun noch die vielfältigen Bezüge von Celans Gedicht zu Hölderlins Patmos, so ergibt sich, dass er Hölderlins Insel und ihrem „Wo aber Gefahr ist, wächst / Das Rettende auch." eine ebenfalls griechisch geprägte Landschaft ganz anderer Art in einem, Gegengedicht ' gegenüberstellt, wie sie von Celan auch sonst bekannt sind 33 .

Wenn Entwurf einer Landschaft eine antike Stätte in Griechenland als Anregung zugrundeliegt, so stellt dies im Werk Celans keineswegs ein überraschendes oder auch nur seltenes Phänomen dar. Die Gedichte Paul Celans berühren mit Bezügen auf antike Autoren und Mythen, aber auch archäologische Stätten und Objekte die Welt

30 Vgl. zum Charakter der Landschaft BAER, Traumadeutung [Anm. 1], p. 238-256; IHRIG, „Landschaftsentwürfe“ [Anm. 1], p. 302-303; MANGER, „Entwurf“ [Anm. 1], bes. p. 350351; SCHELlENBERGER, „Gletscherstuben“ [Anm. 1], p. 350-351.

Z. Herbert, Im Vaterland der Mythen. Griechisches Tagebuch (Herausgegeben von K. DEDECIUS), Frankfurt/Main, 2001, p. 21.

32 Dies wird von der Bedeutung des Gedichts Entwurf einer Landschaft von Carl Einstein nicht beeinträchtigt, die IHRIG, „Landschaftsentwürfe“ [Anm. 1] betont; vgl. MANGER, "Entwurf" [Anm. 1], p. 355-356. Zur häufigen Verwendung von Zitaten durch Celan vgl. D. Goltschnigg, „Das Zitat in Celans Dichtergedichten“, in STrelka, Psalm [Anm. 6], p. 50-63; J. RYAN, „Die 'Lesbarkeit der Welt' in der Lyrik Paul Celans“, in STRELKA, Psalm [Anm. 6], p. 14-21, bes. p. 18-19.

33 Zu Celans Entwurf einer Landschaft und Hölderlins Patmos vgl. K. Manger, „Paul Celans poetische Geographie“, in STRELKA, Psalm [Anm. 6], p. 143-170, bes. p. 155-160; MANGER, „Entwurf“ [Anm. 1], p. 351. Zitat aus Patmos nach SCHMIDT, Hölderlin [Anm. 18], p. 350. - „Gegengedichte“: vgl. RYAN, „Lesbarkeit“ [Anm. 32], p. 20. 
der Antike vielmehr in vielfältiger Weise ${ }^{34}$. So hat auch R. Böschenstein schon zu Recht darauf hingewiesen, dass antike Motive einen „zentralen Part jener intertextuellen Züge ausmachen, welche die Zeichenwelt, in der Celan lebte und schrieb, konstituieren 35“. Eine Durchsicht der Gedichte Celans auf antike Motive - zunächst außerhalb archäologischer Zusammenhänge - lässt dabei rasch einige Schwerpunkte erkennen, die fast ausschließlich der griechischen Antike angehören 36. Dabei lassen sich bloße Erwähnungen, längere Textpartien oder auch ganze Gedichte nennen, die thematisch der Antike verpflichtet sind.

Am häufigsten werden bei Celan griechische Autoren und Philosophen genannt oder zitiert, immer wieder auch nur auf sie angespielt ${ }^{37}$ : Allen voran steht dabei Homer, dessen deutscher Übersetzung durch Johann Heinrich Voß zahlreiche Wörter und Wendungen entnommen sind 38 ; auf eine andere Übersetzung, nämlich diejenige Pindars durch Hölderlin, wird gleich in mehreren Gedichten Bezug genommen, wobei für Celan sicherlich Hölderlin und nicht Pindar im Vordergrund des Interesses gestanden hat ${ }^{39}$. Mit Demokrit, Empedokles, Parmenides und Zenon von Elea begegnen

$34 \mathrm{Zu}$ Celan und der klassischen Antike vgl. Baumann [Anm. 13], p. 102-103; BollaCK 2006, passim, bes. auch p. 279-299; R. BÖSCHENSTEIN, „Nach der Flut. Zum Gedicht 'Deukalion und Pyrrha'“, Celan-Jahrbuch 3 (1989), p. 7-22; K. MANGER, „Mit wechselndem Schlüssel. Zur Dichtung Paul Celans“, Euphorion 75 [1981], p. 444-473). Vgl. allgemein auch B. SEIDENSTICKER, „Zur Antikerezeption in der deutschen Literatur nach 1945“, Gymnasium 98 (1991), p. 420-459; B. SEIDENSTICKER - P. HABERMEHL (Hrsg.), Unterm Sternbild des Hercules. Antikes in der Lyrik der Gegenwart, Frankfurt/Main 1996 m. Lit.; H. SichtermanN, Kulturgeschichte der klassischen Archäologie, München, 1996, p. 331-372 sowie J. MEYer (Hrsg.), Antike in Sicht. Strandgut aus dem Deutschen Literaturarchiv, marbacher magazin 107, Marbach, 2004.

35 BösChEnSTEIN, „Flut" [Anm. 34], p. 7.

$36 \mathrm{Zu}$ verschiedenen Begriffen, die auf die Antike zurückgehen, vgl. (Nr. 66-70).

37 Vgl. (Nr. 1-14).

38 (Nr. 7). Jean Bollack wies darauf hin, dass Celan in Paris W. ScHADEWALDT, Von Homers Welt und Werk, Leipzig, 1944. Stuttgart ${ }^{4}, 1965$ benutzt und sich Notizen gemacht hat (Bollack 2006, p. 279). Dazu merkt Bollack in Anm. 2 an, Schadewaldt sei zur Zeit der Entstehung des Buches „Mitglied der NSDAP und Dekan der Philosophischen Fakultät Leipzig" gewesen. Schadewaldt war in der Tat bis zur Annahme des Rufes nach Berlin zum WS 1941/42 Dekan in Leipzig, jedoch nach H. FLASHAR, „Biographische Momente in schwerer Zeit", in TH. A.SzLEZÁK (Hrsg.), Wolfgang Schadewaldt und die Gräzistik des 20. Jahrhunderts, Hildesheim/Zürich/New York, 2005, p. 167 nie Mitglied der NSDAP; vgl. auch J. Malitz, „Klassische Philologie“, in E. Wirbelauer (Hrsg.), Die Freiburger Philosophische Fakultät 1920-1960. Mitglieder - Strukturen - Vernetzungen, Freiburg/München, 2006, p. 304-315, bes. p. 314 m. Lit.; zur Parteimitgliedschaft vgl. p. 312 Anm. 32: Schadewaldt ist „anders als Heidegger... nicht in die Partei eingetreten.“

39 (Nr. 9). Vgl. dazu B. BÖsChENSTEIN, „Hölderlin und Celan“, Hölderlin-Jahrbuch 23 (1982/83), p. 147-155; BOLLACK 2006, p. 167-217. 
dann Vertreter der vorsokratischen Philosophen recht häufig ${ }^{40}$, wobei Celan wie bei Pau, nachts mitunter von ganz objektbezogenen gedanklichen Verbindungen ausging, hier einem Schildkrötenpanzer im Schloss der Pyrenäenstadt ${ }^{41}$ :

Pau, nachts

Die Unsterblichkeitsziffer, von Heinrich

dem Vierten in

den Schildkrötenadel gewiegt,

höhnt eleatisch

hinter sich her.

Weitere Philosophen wie Platon oder Plotin bleiben seltene Anregung ${ }^{42}$, was ebenso für den Fabeldichter Äsop ${ }^{43}$ oder den Redner Demosthenes ${ }^{44}$ gilt. Anders verhält es sich dagegen mit Hippokrates und den unter dem Namen des Hippokrates versammelten Berichten über Heilungen von Krankheiten, die Celan mehrfach zu Anspielungen, aber auch längeren Zitaten veranlasst haben, was sich zu der großen Bedeutung von Krankheit für Celan fügt ${ }^{45}$; Anknüpfungspunkte an das eigene Erleben boten ihm daneben vor allem die Region des Schwarzen Meeres und die damit verbundenen Mythen ${ }^{46}$. Weiter nehmen griechische Gottheiten und Heroen wie Athena, Dionysos oder Odysseus bei Celan breiten Raum ein, jedoch ohne dass sich hier ausgesprochene Vorlieben feststellen lassen ${ }^{47}$. In literarischer und religiöser Tradition steht schließlich das seit dem alexandrinischen Dichter Kallimachos

40 (Nr. 3; 5; 8; 14). Für Empedokles waren Arbeiten des Gräzisten Jean Bollack von Bedeutung, mit dem Celan befreundet war; vgl. BOLlaCK 2000, p. 289; BOlLACK 2006, p. 301-314. - In Gegenlicht hat Celan eine berühmte Sentenz zitiert, der ein Gedanke des Heraklit zugrundeliegt: „Alles fließt': auch dieser Gedanke, und bringt er nicht alles wieder zum Stehen?"“; P. CELAN, Gegenlicht (Die Tat 12. März 1949), in: B. AllemanN St. Reichert (Hrsg.), Paul Celan, Gesammelte Werke, Dritter Band. Gedichte III. Prosa. Reden, Frankfurt/Main, 1986, p. 165. Heraklit war auch wichtiges Gespächsthema: vgl. Baumann, Celan [Anm. 13], p. 102-103.

41 Entstanden 23.10.-30.10.1965 in Pau und Paris, publiziert 1968 in Fadensonnen; (Nr. 14). - Zum Verständnis des Gedichts vgl. Wiedemann 2003, p. 756 mit Ausführungen zu dem im Schloss von Pau als Wiege Heinrichs IV. benutzten Schildkrötenpanzer, der Ermordung Heinrichs in Pau sowie dem Paradox des Zenon von Elea, nach dem Achilleus im Wettlauf eine vor ihm startende Schildkröte niemals einholen kann, da sich die beide trennende Strecke immer nur halbiere, so dass stets ein Vorsprung bleibt. (Nr. 10;11).

43 (Nr. 2).

44 (Nr. 4).

45 (Nr. 6); vgl. BOLLACK 2000, p. 146.

46 (Nr. 16; 22; 43; 46; 63; 65); vgl. u. 106-110.

47 (Nr. 15-34). 
gerühmte Sternbild der „Locke der Berenike“, das auf ein Haaropfer der ptolemäischen Königin Berenike zurückgeht, und bei Celan mehrfach vorkommt 48 .

Archäologische Stätten und antike Orte werden bei Celan nicht sehr häufig genannt, doch können sie wie der Bereich des Schwarzen Meeres existentielle Bedeutung gewinnen ${ }^{49}$. Eine herausragende Rolle nimmt dabei Cerveteri ein, das in zwei Gedichten ein zentrales Thema ist; Mittags wurde bereits zitiert ${ }^{50}$. Eine erschreckende Verwandlung des antiken Ortes vollzieht sich in Die Ewigkeit 51 :

Die EWIGKeIT altert: in

Cerveteri die

Asphodelen

fragen einander weiß.

Mit mummelnder Kelle, aus den Totenkesseln, übern Stein, übern Stein, löffeln sie Suppen in alle Betten und Lager.

Die „Asphodelen“ werden bereits in der Antike als Pflanzen auf Wiesen, aber eben auch in der Unterwelt genannt ${ }^{52}$. Doch werden sie in Die Ewigkeit mit „öffeln sie Suppen / in alle Betten / und Lager" zugleich in die Situation des Konzentrationslagers versetzt 53: ein verwandtes Ineinandergreifen von Antike und dem Holocaust findet

48 (Nr. 35); vgl. auch P. CELAN, Edgar Jené und der Traum vom Traume, Wien, 1948, in Allemann-Reichert, Celan [Anm. 40], p. 159: „Ungläubig auch heute noch, wo doch das Haar der Berenike schon so lange unter den Sternen hängt“. - Weiteres Sternbild: Orion (Nr. 36).

49 (Nr. 39-47); zum Schwarzmeergebiet vgl. u. 106-110. - Für Paris verwendete Celan in einem Brief an Franz Wurm den antiken Namen Lutetia mitsamt einem Wortspiel zu „gallisch“: „Ich selbst muß die Hundstage gallisch erweise in Lutetia verbringen..."; Brief vom 5.5.1967, vgl. B. Wiedemann in Verbindung mit Franz Wurm (Hrsg.), Paul Celan - Franz Wurm. Briefwechsel, Frankfurt/Main, 1995, p. 64-65, Nr. 40 (Zitat ebda. p. 65).

50 Vgl. o. 94-95.

51 Entstanden 11.4.1967, publiziert 1968 in Fadensonnen (Nr. 41); vgl. WiedeMANN 2003, p. 772; auch erwähnt bei PÖGGELER, Kunst [Anm. 1], p. 346.

52 R. WAGNER, s. v. „asphodelos“, in RE, II, 1896, col. 1730-1733.

53 Vgl. auch H. MAYER, Ein Deutscher aufWiderruf. Erinnerungen Band II, Frankfurt/Main, 1984, p. 322-323. - Die „Suppe“ als existentielle Erfahrung wird in vielen autobiographischen Berichten beschrieben: Vgl. V. FranKL, ... trotzdem Ja zum Leben sagen. Ein Psychologe erlebt das Konzentrationslager, München ${ }^{11}$ 1992, p. 53-56, bes. p. 54; 80; P. LEVI, Ist das ein Mensch?, München, 1992, p. 36; 87-90; 148; 155-156; J. SEMPRUN, Was für ein schöner Sonntag!, Frankfurt/Main, 1984, p. 223-225. - Zur Thematik des Holocaust bei Celan vgl. BOLLACK 2000, p. 67-71; 138-141; 161-184; B. MurdOCH, 
sich auch in Denk Dir mit seinem Vers „Der Moorsoldat von Massada 54“. Wie unterschiedlich Celan historische Themen aktualisierte und so neu gestalten konnte, wird am Troja seines Gedichts Sperrtonnensprache deutlich 55:

SPERRTONNENSPRACHE, Sperrtonnenlied.

Die Dampfwalze wummert

die zweite

Ilias

ins aufgerissene

Pflaster,

sandgesäumt

staunen die alten

Bilder sich nach, in die Gosse,

ölig verbluten die Krieger

in Silberpfützen, am Straßen-

rand, tuckernd,

Troja, das staubbekrönte,

sieht ein.

Dass sich die Thematik gewaltsamer Ereignisse hier ebenso zeigt wie in der vulkanischen Beschaffenheit von Entwurf einer Landschaft und der misshandelten "Tierstirn“ muss kaum mehr als erwähnt werden und stimmt hier ebenso zum angeschlagenen antiken Thema wie dort. Es mag dabei noch erwähnt sein, dass Celan zwar Cerveteri kannte, andere bei ihm genannte antike Orte wie Babylon, Massada oder Troja jedoch sicherlich nie besucht hat 56 .

„Transformations of the Holocaust. Auschwitz in Modern Lyric Poetry“, Comparative Literature Studies 11 (1974), p. 132-135; SAMUELS, Holocaust Visions [Anm. 1].

Entstanden 7.6. - 13.6.1967 (Nr. 44); vgl. I. ShMUeli - Th. SparR (Hrsg.), Paul Celan Ilana Shmueli. Briefwechsel, Frankfurt/Main, 2004, p. 7, Nr. 1 Anm. 2; WiedEMANN 2003, p. 790 (jeweils mit Hinweisen zum Lied „Wir sind die Moorsoldaten“, der Belagerung von Massada und zum Sechstagekrieg); vgl. auch BAER, Traumadeutung [Anm. 1], p. 259-272; BollaCK 2000, p. 206-207; BollaCK 2006, p. 23; SAMUels, Holocaust Visions [Anm. 1], p. 73-75. Während seiner Reise nach Israel im Jahre 1969 ließ Celan einen geplanten Besuch in Massada aus; vgl. Felstiner, Celan [Anm. 1], p. 340. Entstanden 1.11.1967, publiziert in Lichtzwang (Nr. 47).

56 Cerveteri kannte Celan seit seinem Aufenthalt 1964 in Rom; vgl. WIEDEMANN 2003, p. 732. Über Rom äußerte sich Celan in einem Brief an Gisèle Celan-Lestrange vom 17.4.1964 nicht sehr enthusiastisch: „Rom macht einen etwas chaotischen Eindruck auf mich, man fühlt sich von seinen Steinen ein wenig angegriffen"; B. BADIOU in Verbindung mit E. Celan (Hrsg.), Paul Celan - Gisèle Celan-Lestrange: Briefwechsel, Anmerkungen übersetzt und für die deutsche Ausgabe eingerichtet von B. WIEDEMANN, Frankfurt/Main, 2001, p. 160 Nr. 182. Zu Massada vgl. Anm. 54. 
Archäologische Objekte wie das mykenische Stierkopfgefäß bleiben in Celans Gedichten eher selten, und ihre Erwähnung beruht mitunter auch auf einer Textgrundlage 57: So erwähnt Celan in In der ewigen Teufe einen „Mischkrug voll blasigen / Hirns 58“, spielt hier jedoch nicht auf ein von ihm gesehenes Objekt, sondern auf ein berühmtes Fragment des vorsokratischen Philosophen Xenophanes an, wie nach B. Wiedemann aus seinen „Lektürenotizen zu Xenophanes aus dem August 1952“ hervorgeht 59: „Denn nun ist ja der Fussboden rein und aller Hände und Becher. Gewundene Kränze legt uns einer ums Haupt, und ein anderer reicht duftende Salbe in einer Schale dar. Der Mischkrug steht da angefüllt mit Frohsinn, auch noch ein anderer Wein ist bereit in den Krügen, der nimmer zu versagen verspricht, ein milder, blumenduftender."

Ähnlich ,unarchäologisch“ dürften auch die „Siebenflöte“ - Syrinx - in Aus den nahen ${ }^{60}$ oder insbesondere der in seiner symbolischen Bedeutung verwendete Pfeil in Der Pfeil der Artemis zu verstehen sein, dem ein ganzes Gedicht gilt 61:

57 (Nr. 59-62). Die Bedeutung von Kunstwerken und Artefakten früher Kulturen bei Celan scheint als eigenständiges Thema bislang nur von PöGGELER, Kunst [Anm. 1], p. 281-375 behandelt worden zu sein. Zeugnisse der Ur- und Frühgeschichte sowie Ägyptens werden im folgenden nicht näher diskutiert; vgl. Le Menhir (Wiedemann 2003, p. 150); Spasmen, V. 8: „Knochenstabritzung“ (WiedemanN 2003, p. 225); Der Geglückte, V. 2: „Mumiensprung" (WIEDEMANN 2003, p. 233); Muschelhaufen, V. 2: „Geröllkeule“ (Wiedemann 2003, p. 275); Streu Ocker, passim (Wiedemann 2003, p. 302); auch erwähnt bei PöGgeler, Kunst [Anm. 1], p. 347-353. Die von PÖGGELER p. 355-356 genannten „Lößpuppen“ des gleichnamigen Gedichtes (WIEDEMANN 2003, p. 337) gehören offenbar in einen anderen Zusammenhang; vgl. B. KLOSE, "Souvenirs entomologiques'. Celans Begegnung mit Jean-Henri Fabre“, in Ch. SHoHAM - B. WiTTE (Hrsg.), Datum und Zitat bei Paul Celan. Akten des Internationalen Paul Celan-Colloquiums Haifa 1986, Jahrbuch für Internationale Germanistik Reihe A. Kongressberichte, Band 21, Bern/Frankfurt (Main)/New York/Paris, 1987, p. 145-146. Im folgenden bleiben auch die Zeugnisse jüdischer Kultur ausgeklammert, die bereits mehrfach erörtert worden sind; vgl. BOLlaCK 2000, p. 191-213; BOLlaCK 2006, p. 127-152; Felstiner, Celan [Anm. 1], passim.

58 Entstanden Paris, 10.10.1965, publiziert 1968 in Fadensonnen (Nr. 59).

59 Wiedemann 2003, p. 753. Es handelt sich um H. Diels - W. KranZ, Die Fragmente der Vorsokratiker, Zürich/Hildesheim ${ }^{18}$, 1989, p. 126-128 Fragment B 1 (Übersetzung). Zur Bedeutung der vorsokratischen Philosophie für Celan vgl. o. 101-102. Der "Mischkrug“ ist eine etwas irreführende Übersetzung für das griechische Wort Krater, ein großes Mischgefäß unterschiedlicher Formgebung.

60 Entstanden Moisville, 1.8. - 4.8.1966 (Nr. 61).

61 Entstanden Czernowitz 1944, publiziert 1946 in Der Sand aus den Urnen (Nr. 60). 
Der pfeil der artemis

Für Alfred Margul-Sperber

Die Zeit tritt ehern in ihr letztes Alter.

Nur du allein bist silbern hier.

Und klagst im Abend um den Purpurfalter.

Und haderst um die Wolke mit dem Tier.

Nicht, daß dein Herz nie Untergang erfuhr

und Finsternis nie deinem Aug befahl...

Doch trägt vom Mond noch deine Hand die Spur.

Und in den Wassern sträubt sich noch ein Strahl.

Wie soll, der über himmelblauen Kies

sich mit den Nymphen drehte, leicht,

nicht denken, daß ein Pfeil der Artemis

im Wald noch irrt und ihn zuletzt erreicht?

Auch die „Kanopen“ in Die Silbe Schmerz sind von der Lektüre Celans - nun wieder von Fachliteratur - angeregt ${ }^{62}$ :

... In Särgen,

Urnen, Kanopen

erwachten die Kindlein

$\mathrm{Zu}$ diesen Versen hat B. Wiedemann auf eine Lesenotiz Celans hingewiesen, die "Lokalformen wie die Kanopen von Chiusi“ betrifft 63. Gemeint ist damit, dass in Chiusi anders als im übrigen Etrurien auch noch nach Ende des 8. Jhs. v. Chr. Brandbestattungen vorgenommen wurden, für die als Aschenurnen Gefäße mit zum Teil anthropomorpher Gestaltung hergestellt wurden; dabei wurden einem als Körper dienenden Gefäß ein plastisch ausgeführter Kopf und Arme angesetzt ${ }^{64}$.

In zwei anderen Gedichten hat Celan mit skythischen Filzschwänen jedoch den visuellen Eindruck einer Grabbeigabe genutzt. Dies zunächst in dem zuerst entstandenen Gedicht Schwanengefahr 65:

SCHWANENGEFAHR,

Lappentaucher-

Bedrohung,

62 Entstanden 16.9.1962, publiziert 1963 in Die Niemandsrose (Nr. 53), zitiert V. 31-33.

63 Vgl. WiEDEMANN 2003, p. 709-710.

64 Vgl. jetzt HaYnes, Etrusker [Anm. 11], p. 130-132; PraYON, Etrusker [Anm. 11], p. 4748.

65 Entstanden Paris, 20.11.1967, publiziert 1970 in Lichtzwang (Nr. 65). 
der Eisbewimperte mit

Kraken-

armen,

du, bekrallter

Jakuten-

Puschkin:

Hei, Chebeldei, Chebeldei.

Noch deutlicher auf die skythischen Filzschwäne bezogen findet sich dasselbe Motiv in Ungewaschen, unbemalt 66 :

UNGEWASCHEN, UNBEMALT,

in der Jenseits-

Kaue:

da,

wo wir uns finden,

Erdige, immer,

ein

verspätetes

Becherwerk geht

durch uns Zerwölkte hindurch,

nach oben, nach unten,

aufrührerisch

flötets darin, mit Narren-

beinen,

der Flugschatten im

irisierenden Rund

heilt uns ein, in der Sieben-

höhe,

eiszeitlich nah

steuert das Filzschwanenpaar

durch die schwebende

Stein-Ikone

66 Entstanden auf dem Flug von Paris nach Berlin, 16.12.1967, publiziert 1971 in Schneepart (Nr. 65). 
Celan hat in Paris wohl gemeinsam mit seinem Sohn Eric die Ausstellung "L'Art russe des Scythes à nos jours" besucht, was ihn zu beiden Gedichten anregte ${ }^{67}$. Skythische Filzschwäne waren auch bei späteren Skythenausstellungen zu sehen (Abb. 5) ${ }^{68}$. Der hier abgebildete $35 \mathrm{~cm}$ lange Schwan stammt mit drei weiteren entsprechend gestalteten Schwänen aus dem bedeutenden Grabhügel (Kurgan) 5 in Pazyryk, dessen Beigaben in das 5. und 4. Jh. v. Chr. datiert werden können ${ }^{69}$. Die Schwäne wurden aus Filz angefertigt und mit Moos ausgestopft; sie sind in ihrer detaillierten Ausführung - der Proportion und dem Höcker auf dem Schnabel - und ihrer farblichen Gestaltung - weißes Gefieder,

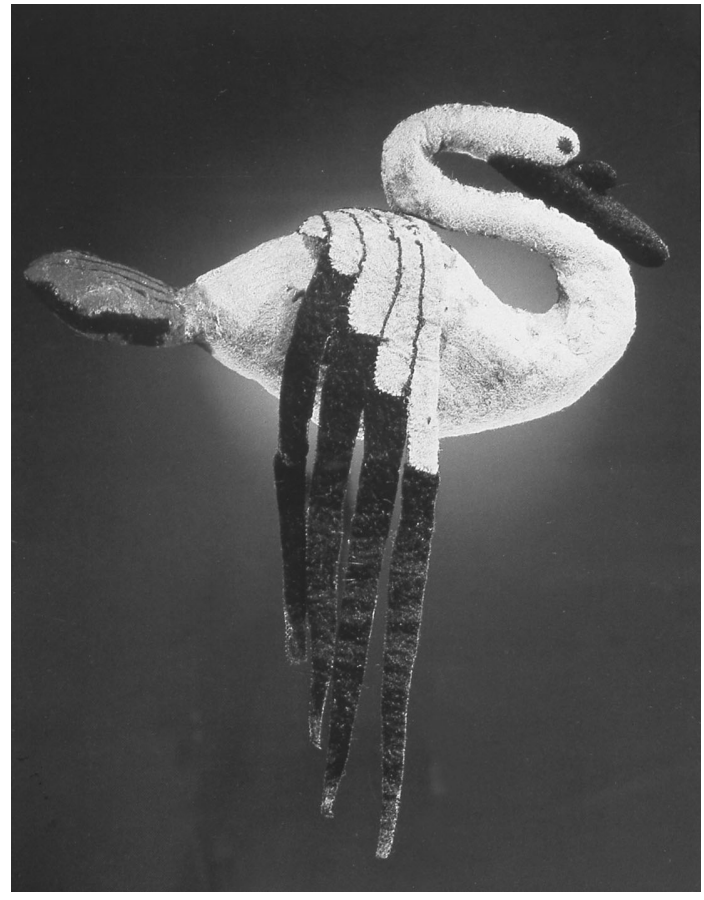

Abb. 5. Skythischer Schwan aus Filz schwarzer Schnabel und Flügelspitzen, rotschwarzer Schwanz und rötliche Beine - dem Naturvorbild getreu nachempfunden. Die vier Schwäne bildeten ursprünglich den Schmuck eines Totenzeltes oder eines Paradewagens; von beidem wurde Reste in der Grabanlage entdeckt. Der außerordentlich reiche Kurgan 5 von Pazyryk ist für seine Textilfunde besonders berühmt, zu dem außer den Filzschwänen ein Filzteppich mit Reiter, ein Knüpfteppich mit Reiterzügen sowie eine in China gefertigte Pferdeschabracke aus Seide gehörten.

Dass gerade ein Werk der skythischen Kultur für Celan bedeutungsvoll werden konnte, wird aus der mehrfachen Erwähnung von Skythen in seinem Werk verständlich 70. So thematisiert das Gedicht Und mit dem Buch aus Tarussa die antike

67

68 Vg. WIEDEMANN 2003, p. 825.

68 Vgl. V. SCHILTZ, Les Scythes et les nomades des steppes. VIII siècle avant J.-C. - Ier siècle après J.-C., L'Univers des Formes, Paris, 1994, p. 264 m. Abb. 197; K. VIERNEISEL (Hrsg.), Gold der Skythen aus der Leningrader Eremitage, Ausstellungskatalog München, 1984, p. 216217, Nr. 129.

69

Zu Pazyryk vgl. SchiLTZ, Scythes [Anm. 68], bes. p. 260-264; 348-350; 427-433; VIERNEISEL, Skythen [Anm. 68], p. 164-218.

70 
Schwarzmeerregion, insbesondere Kolchis, und die Argonauten, wobei dann auch von „skythisch / zusammengereimt / im Takt 71 “ die Rede ist. Weiter ist ein Skythe Hauptthema von Sichtbar-unsichtbar ${ }^{72}$ :

Sichtbar-unsichtbar:

ein blauweißer Skythe,

zebragewandet, beritten,

rotdeutsch,

schwarzwelsch,

vor sich her-

atmend

den Einen

Pfeil.

Dass der Skythe als „ein blauweißer Skythe, / zebragewandet“ beschrieben und ein „Pfeil“ erwähnt wird, entspricht antiken Vorstellungen. So zeigen Darstellungen Skythen, die für ihre Künste mit Pfeil und Bogen berühmt waren, stets mit bunt gemusterten Gewändern ${ }^{73}$. Die Skythen waren Celan nun aus verschiedenen Gründen nahe ${ }^{74}$. Seine Heimatregion, die Bukowina 75 ist nicht allzu weit vom Schwarzen Meer - einem der skythischen Gebiete - entfernt, doch ebenso auch der Deportationsund Todesort seiner Eltern ${ }^{76}$ : doch wurde in diesem Zusammenhang auch an die literarische Gruppe Die Skythen erinnert ${ }^{77}$. Aufgrund ihrer Herkunft besaßen die skythi-

71 Entstanden Moisville - Paris, 20.9.1962, publiziert 1963 in Die Niemandsrose (Nr. 64); zum Pontosgebiet, den Argonauten und Iason vgl. o. Anm. 46. Zu „skythisch“ vgl. J. Lehmann, „Und mit dem Buch aus Tarussa“, in J. Lehmann (Hrsg.), Kommentar zu PAUL CELANs „Die Niemandsrose“" Heidelberg, 1997, p. 360-361.

72 Entstanden 28.12.1966, publiziert aus dem Nachlass (Nr. 63). Zum Gedicht vgl. BOLLACK 2000, p. 199-202.

73 Tracht: Vgl. B. BÄBLER, Fleissige Thrakerinnen und wehrhafte Skythen, Beiträge zur Altertumskunde Band 108, Stuttgart/Leipzig, 1998, p. 173-174. Die Unterscheidung von anderen ,orientalisch' gekleideten Barbaren fällt nicht immer leicht; vgl. A. SCHOLL, „Der ,Perser' und die 'skythischen Bogenschützen' aus dem Kerameikos“, Jahrbuch des Deutschen Archäologischen Instituts 115 (2000), bes. p. 99-102.

74 Vgl. LeHMANN, „Tarussa“ [Anm. 71], p. 360-361.

75 Zum Leben in Czernowitz vgl. die Erinnerungen von I. Shmueli, in I. SHMUELI Th. Sparr (Hrsg.), Paul Celan - Ilana Shmueli. Briefwechsel, Frankfurt/Main, 2004, p. 155-163; Z. YAVETZ, Erinnerungen an Czernowitz, München, 2007.

76 Vgl. BollaCK 2006, p. 55-71; Felstiner, Celan [Anm. 1], p. 25-69.

77 Vgl. J. LEHMANN, „Dichten heißt immer unterwegs sein'. Literarische Grenzüberschreitungen am Beispiel Paul Celans“, Arcadia 28 (1993), p. 124-125; LEHMANN, „Tarussa“ [Anm. 71], p. 360-361. 
schen Schwäne demnach für Celan eine sehr grundsätzliche und eine biographisch geprägte Bedeutung.

Dass sich die genannten archäologischen Orte und Zeugnisse - Cerveteri, Mykene, das Stierkopfgefäß, die Filzschwäne - so häufig auf Gräber und Grabbeigaben beziehen ${ }^{78}$, ist sicher kein Zufall, sondern stimmt mit der nicht seltenen Nennung von Bestattungsformen der italischen Welt sowie der westeuropäischen Bronzezeit und des Alpenraums ${ }^{79}$, aber auch einem Gedicht wie Kenotaph oder Im Schlangenwagen mit seiner Unterweltslandschaft überein, was Celans Präferenz für die Thematik bezeugt ${ }^{80}$. Und dass schließlich archäologische Objekte in den Gedichten Celans eher selten thematisiert werden, trifft sich mit der ebenfalls nicht sehr großen Häufigkeit von Kunstwerken anderer Epochen, die gleichwohl für ganze Gedichte prägend sein können ${ }^{81}$ : So ist auch die Bildkunst und sind archäologische Zeugnisse für Celan immer wieder entscheidende Anregung gewesen - wie noch mehr die von ihm gelesenen oder auch nur benutzten Bücher.

\author{
Matthias STEINHART \\ Staatliche Antikensammlunger un Glyptotheke \\ Meisenstraße, 10 \\ D-80333 München \\ steinhart@antike-am-koenigsplatz,mwn;de
}

78 (Nr. 48-58).

79 Italisch: (Nr. 55). - Bronzezeit, Alpenraum: Heute und morgen (WIEDEMANN 2003, p. 95); vgl. TOBIAS, Nature [Anm. 1], p. 27-28. - Windgerecht, V. 17: „brandbestattet“ (WIEDEMANN 2003, p. 101, vgl. p. 655: Hinweis auf Lesenotiz zu Brandbestattungen in der westeuropäischen Bronzezeit). - Was näht*, V. 15: „Tumuli, Tumuli“ (WIEDEMANN 2003, p. 318).

80 (Nr. 54); zu antiken Kenotaphen vgl. D. C. KurTZ - J. BoArdman, Thanatos. Tod und Jenseits bei den Griechen, Mainz, 1985, passim, bes. p. 303-316. - Im Schlangenwagen (Nr. 48).

81 Rembrandt: Einkanter. WiEDEMANN 2003, p. 336. In diesem Zusammenhang ist auf verschiedene Bilder verwiesen worden; vgl. TH. BuCK, "Mit entsichertem Herzhirn“, Aachen, 2000, p. 86-104; Felstiner, Celan [Anm. 1], p. 329; R. Peuss, „Rembrandts Celan“, Celan-Jahrbuch 3 (1989), p. 47-98; PöGgeler, Kunst [Anm. 1], p. 330-334; Wiedemann 2003, p. 853; W. WÖGERBAUER, „Drei Skizzen zu Bildgedichten Paul Celans“, Celan-Jahrbuch 7, (1997/98), p. 166-170. - Gericault, Cheval pommelé effrayé par la foudre: Blitzgeschreckt. WiedemanN 2003, p. 284; vgl. PÖGGELER, Kunst [Anm. 1], p. 320; WÖGERBAUER, „Skizzen“ [Anm. 81], p. 170-172. - Vincent van Gogh, Weizenfelder mit Raben: Unter ein Bild. WiEDEMANN 2003, p. 94, vgl. p. 646-647; vgl. BucK, „Herzhirn“ [Anm. 81] p. 77-86; Th. BuCK, „Rabenüberschwärmt'. Lyrische Fortschreibungen eines Bildmotivs von Millet und van Gogh bei Paul Celan und Wulf Kirsten“, Celan-Jahrbuch 8 (2000/2001), p. 279-315; PÖGGELER, Kunst [Anm. 1], p. 321330; WÖGERBAUER, „Skizzen“ [Anm. 81], p. 173-175. - Bilder Chagalls waren eine 


\section{Anhang: Die klassische Antike in den Gedichten Paul Celans}

Der folgende Anhang versteht sich als Übersicht zu eindeutigen und vermuteten Bezügen Celans auf die Antike (die an dieser Stelle auch nicht durchgehend diskutiert werden konnten) und damit als Anregung für die weitere Beschäftigung mit dieser Thematik ${ }^{82}$. - Gliederung: I. Autoren, Philosophen. II. Religion und Mythos: a. Mythos. b. Sternzeichen. c. Weissagung. III. Orte und Landschaften. IV. Sachkultur. V. Grab und Unterwelt: a. Gräber und Grabsitten. b. Unterwelt und Jenseitsvorstellungen. c. Pappeln. VI. Skythen. VII. Begriffe antiken Ursprungs. VIII. Begriffe aus der altertumswissenschaftlichen Literatur. Die in der DA aufgeführten Gedichte Celans sind mit einem Asterisk gekennzeichnet.

\section{Autoren, Philosophen}

1. Aristoteles: Die Sämlinge* (WIEDEMANn 2003, p. 246), V. 1: „causa secunda“; der Bezug wird in der DA vorgeschlagen.

2. Äsop: Es wächst, V. 2 (WiEDEMANN 2003, p. 533).

3. Demokrit: Engführung, V. 71: „wir lasens im Buche, war / Meinung“ (Wiedemann 2003, p. 115, vgl. p. 668-669. - Schliere (WIEDEMANn 2003, p. 96).

4. Demosthenes: Sibirisch V. 12-13: „mit deinem / weißen Kiesel im Mund“ (WiedemanN 2003, p. 144, vgl. p. 691). Zur Nachtordnung, V. 11-12: „Weißkies- / stotterer“ (WIEDEMANN 2003, p. 324).

5. Empedokles: Ortswechsel (Wiedemann 2003, p. 366); zur Deutung vgl. Bollack 2006, p. 301-314.

6. Hippokrates: Fortgewälzter*, V. 4 (WIEDEMANN 2003, p. 257). - Haut ma**, V. 4-14: „deine Zunge ist rußig, / dein Harn schwarz, / wassergallig dein Stuhl... schmiegst dich ins Ziegenfell“ (WIEDEMANN 2003, p. 259, vgl. p. 788-789). - In Echsen-, V. 1-7: „IN ECHSEN- / häute, Fall- / süchtige“ (WIEDEMANN 2003, p. 319, vgl. p. 836).

7. Homer: Das Nichts, V. 10: „im Ungeschiednen“ (WIEDEMANN 2003, p. 364, vgl. p. 795). Vgl. Sprich auch Du, V. 5 (vgl. Wiedemann 2003, p. 795). Einbruch, V. 1: „EINBRUCH des Ungeschiedenen / in deine Sprache“ (WIEDEMANN 2003, p. 268, vgl. p. 795). - Füll die Ödnis, V. 2: „Salzflut“ (WIEDEMANn 2003, p. 267, vgl. p. 795). - Sperrtonnensprache* (Wiedemann 2003, p. 299), V. 4: „Ilias“. - Über die Köpfe, V. 2: „hinweggewuchtet“

Inspiration bei: Hüttenfenster. WIEDEMANN 2003, p. 157-159, vgl. p. $708 \mathrm{zu}$ „Witebsk“; vgl. zudem BOLLACK 2000, p. 226. - In einen verwandten Zusammenhang gehören das Gedicht Bei Brancusi, zu zweit (WIEDEMANN 2003, p. 280, vgl. p. 805) sowie Celans Essay zu den Bildern des befreundeten Surrealisten Edgar Jené (CELAN, Jené [Anm. 48], p. 155161; vgl. Felstiner, Celan [Anm. 1], p. 83-84). Eine Besonderheit bildet die Zusammenarbeit von Gisèle Celan-Lestrange und Paul Celan; vgl. Baumann, Celan [Anm. 13], p. 45-46; Felstiner, Celan [Anm. 1], p. 174; 279-284; 297. - Zur Bedeutung der bildenden Kunst für Celan vgl. auch BAumanN, Celan [Anm. 13], p. 47-48; 123-124. $\mathrm{Zu}$ prähistorischen Monumenten und Ägypten vgl. Anm. 57. 
(WIEDEMANn 2003, p. 266, vgl. p. 794). - Weihgüsse, V. 1: „WEIHGÜSSE“ (WIEDEMANN 2003, p. 493, vgl. p. 944-945). - Wirfst Du, V. 3: „Ankerstein“; V. 6: „Unbändigen“; V. 8: „Türstein“; V. 9: „Unbezwungene Zelt“ (WIEDEMANN 2003, p. 267, vgl. p. 794). 83

8. Parmenides: Grabschrift für François (Wiedemann 2003, p. 71); zur Deutung vgl. FelSTiner, Celan [Anm. 3], p. 108. - Mit wechselndem Schliussel (WiEDEMANn 2003, p. 74, vgl. p. 632) - Zu beiden Händen, V. 20-21: „Das / Selbe“ (WiedEMANn 2003, p. 129, vgl. p. 676).

9. Pindar: Ars Poetica 62, V. 11-12: "was man zum Reimen braucht, nach / Pindar" (WIEDEMANN 2003, p. 474, vgl. p. 934). - Ich trink Wein*, V. 2-5: ,und zackere an / der Königszäsur / wie Jener / am Pindar" (Wiedemann 2003, p. 363, vgl. p. 875-876).

10. Platon: Den verkieselten Spruch*, V. 11-12: „der Eine / Hauch“ (WIEDEMANn 2003, p. 201; in der DA wird ein Bezug auf Platon oder Plotin vorgeschlagen). - Einmal ${ }^{*}$, V. 6: „Eins und Unendlich" (WIEDEMANN 2003, p. 214; in der DA wird das Zitat auf Platon, Philebos 18 a/b zurückgeführt). - Zur Rechten, V. 3-4: „außer- / himmlischen Ort“ (WIEDEMANN 2003, p. 242, vgl. p. 768); s. auch (Nr. 70).

11. Plotin: Den verkieselten Spruch", V. 11-12: „der Eine / Hauch“ (WIEDEMANn 2003, p. 201; in der DA wird ein Bezug auf Platon oder Plotin vorgeschlagen).

12. Sokrates: Am Brunnen, V. 12: „Der Schierling streift mir träumerisch die Knie“ (WIEDEMANN 2003, p. 14, vgl. p. 584).

13. Xenophon: Anabasis* (Wiedemann 2003, p. 147-148). Celan besaß ein Exemplar von Xenophons Anabasis, was den Bezug auf diesen Autor nahelegt; s. auch (Nr. 70).

14. Zenon: Pau, nachts*: „Unsterblichkeitsziffer... Schildkrötenadel... eleatisch“: Anspielung auf das Paradox des Zenon (Wiedemann 2003, p. 226, vgl. p. 756).

\section{Religion und Mythos}

a. Mythos

15. Quellpunkte*, V. 3: „göttergewärtig“(WIEDEMANN 2003, p. 304).

16. Argonauten: Und mit dem Buch aus Tarussa (WIEDEMANN 2003, p. 164-166).

17. Artemis: Der Pfeil der Artemis* (WIEDEMANN 2003, p. 17).

18. Athena: Wenn ich nicht weiß, nicht weißs, V. 12-16: „der Jüdin / Pallas / Athene / in die behelmten Ovarien gespritzt" (WIEDEMANN 2003, p. 238).

19. Deukalion und Pyrrha: Spät und tief, ursprünglicher Titel: Deukalion und Pyrrha* (WiedeMANn 2003, p. 38, vgl. p. 605).

20. Dionysos: Herbst*, V. 5: „Verteilt ist der sündige Efeu“ (Wiedemann 2003, p. 422; in der DA wird ein Bezug auf Dionysos vermutet). - Müdigkeit*, V. 9: „Rausch und Rebe“

83 Auszuscheiden ist Die Irin*, V. 9-10: „augenfingrige / Ferne“; WIEDEMANN 2003, p. 290291. In der DA wird ein Bezug auf Homers „rosenfingrige Eos“ (Voß) angenommen, doch vertritt KLOSE, „Fabre“ [Anm. 57] p. 124 eine überzeugende Ableitung von der bei Celan mehrfach vorkommenden Gottesanbeterin. 
(Wiedemann 2003, p. 388; in der DA wird ein Bezug auf Dionysos vorgeschlagen). - Zu Haut Mal, zu dem in der DA ein Bezug auf Dionysos angenommen wird, vgl. (Nr. 6).

21. Hephaistos: In der Luft*, V. 44-46: „... darüber / der Klumpfuß der Götter herüber- I gestolpert kommt“ (WIEDEMANN 2003, p. 166-167, vgl. p. 717).

22. Iason: Talglicht*, V. 2: "Jason wirft nun mit Schnee“ (WIEDEMANN 2003, p. 29).

23. Ilioupersis: Sperrtonnensprache* (WIEDEMANN 2003, p. 299).

24. Kalydonischer Eber: In Gestalt eines Ebers (WiEDEMANN 2003, p. 69, vgl. p. 627: Celan bezog den Eber fragend auf den kalydonischen Eber).

25. Kentauren: Hammerköpfiges*, V. 10: „kentaurisch“ (WIEDEMANn 2003, p. 191).

26. Kraniche (Gegner der Pygmäen): - Am letzten Tor (Wiedemann 2003, p. 20, vgl. p. 590) - Bei Tag (Wiedemann 2003, p. 151, vgl. p. 698); zur Deutung vgl. Bollack 2006, p. 26.

27. Mercur: Warum aus dem Ungeschöpften*, V. 7: „Merkurius“ (WIEDEMANN 2003, p. 326; in der DA wird ein Bezug auf den antiken Gott angenommen; WIEDEMANN 2003, p. 840 verweist auf die alchimistische Bezeichnung für Quecksilber).

28. Neptun: Hafen*, V. 60: „neptunische Sünde“ (WiEdemanN 2003, p. 190).

29. Nymphen: Der Pfeil der Artemis*, V. 10 (WIEDEMANn 2003, p. 17).

30. Odysseus: Die Gauklertrommel*, V. 4: „die Odysseus, mein Affe, nach Ithaka klettert“ (Wiedemann 2003, p. 192). Die Nacht, V. 8: „und Odysseus nicht folgt auf der Irrfahrt“ (WIEDEMANN 2003, p. 437).

31. Orion: -> Sternzeichen.

32. Orpheus: Aber, V. 14: „die Schwäne“ (WIEDEMANN 2003, p. 106, vgl. p. 659 mit Hinweis auf Anstreichung bei Platon, Der Staat. Seele des Orpheus in einen Schwan verwandelt).

33. Polyphem: Abzählreime*, V. 10: „Polikarp und Polyphem“ (WiEdemanN 2003, p. 121).

34. Tellus: Huhediblu*, V. 33 (WIEDEMANn 2003, p. 156).

\section{b. Sternzeichen}

35. Locke der Berenike: Hinausgekrönt*, V. 6: „Berenikes Haupthaar, auch hier...“. (WiEdemann 2003, p. 154). - Sternenlied*, V. 20: „Dein Haar vom Glanz aus Berenikes Haar“ (Wiedemann 2003, p. 424). s. auch Anm. 47.

36. Orion: Der Jäger, V. 1: „Im Auge, dem der Orion erlosch“ (WIEDEMANN 2003, p. 425). Dunkles Aug im September, V. 10-11: „Hoffnung auf Orions / baldige Rückkunft...“ (WIEDEMANN 2003, p. 33).

\section{c. Weissagung}

37. Auguren: Es ist alles anders*, V. 28: „Auguren“ (WIEDEMANn 2003, p. 162). - Windmühlen, V. 28 (Wiedemann 2003, p. 162-163); in der DA wird zu V. 70 „Alba“ ein Bezug auf Latium erwogen, doch handelt es sich um einen Fluß, mit WiedEMANN 2003, p. 713 um die Elbe.

38. Sibyllen: Give the word*, V. 7: „(Sipheten und Probyllen sind dabei)“, (Wiedemann 2003, p. 208). - Zeitlücke, V. 3: „sibyllengrau“ (WIEDEMANN 2003, p. 490). 


\section{Orte und Landschaften}

Alba: s. Nr. 37.

39. Akra: Ein Lied in der Wüste (WIEDEMANN 2003, p. 27).

40. Babylon, Gärten der Semiramis: Die Gärten*, V. 12 (WIEDEMANN 2003, p. 417).

41. Cerveteri: Die Ewigkeit* (Wiedemann 2003, p. 245). - Mittags (Wiedemann 2003, p. 187188).

42. Ithaka: Die Gauklertrommel*, V. 4: „die Odysseus, mein Affe, nach Ithaka klettert“ (WIEDEMANN 2003, p. 192).

43. Kolchis: Und mit dem Buch aus Tarussa*, V. 80 (WIEDEMAnN 2003, p. 165); vgl. aber auch LEHMANN, „Tarussa“ [Anm. 71], p. 365-366.

44. Massada: Denk Dir, V. 2: „Der Moorsoldat von Massada“ (Wiedemann 2003, p. 262).

45. Mykene: Entwurf einer Landschaft (WIEDEMANN 2003, p. 107-108).

46. Schwarzes Meer: Aschenglorie*, V. 4: „Pontisches Einstmals“ (WIEDEMANN 2003, p. 198). s. auch Argonauten. Kolchis. Skythen.

47. Troja: Sperrtonnensprache* (WIEDEMANn 2003, p. 299).

\section{Grab und Unterwelt}

a. Unterwelt und Jenseitsvorstellungen

48. Im Schlangenwagen (WIEDEMANN 2003, p. 179).

49. Die Ewigkeit*, V. 3 (Wiedemann 2003, p. 245); in der DA wird eine Verbindung zu den Asphodelen der Unterwelt vermutet.

50. Erratisch*, V. 10-11: „Seele /... im Äther“ (WIEDEMAnN 2003, p. 138; in der DA wird auf den Bezug zu antiken Vorstellungen hingewiesen.

51. Les Globes* (WIEDEMANN 2003, p. 155; in der DA wird ein Bezug auf antike Jenseitsvorstellungen vermutet).

\section{b. Gräber und Grabsitten}

52. Hockergrab: Heute und morgen (Wiedemann 2003, p. 95); einen Bezug auf Hockergräber sieht TOBIAS, Nature [Anm. 3], p. 28-29.

53. Kanopen: Die Silbe Schmerz, V. 32 (WIEDEMANn 2003, p. 159-160).

54. Kenotaph: Kenotaph* (Wiedemann 2003, p. 84).

55. Pozzo-Gräber: Brunnen-, V. 1 f.: „BRUNNEN- / artig / ins Verwunschne vertieft“ (WIEDEMANN 2003, p. 294, vgl. p. 819). - Brunnengräber im Wind, V. 10-11: „Gräber- I brunnen“ (WIEDEMANN 2003, p. 316).

56. Rundgräber: Entwurf einer Landschaft (WIEDEMANN 2003, p. 107-108). - Mittags (WIEDEMANN 2003, 187-188).

57. Schachtgrab: Müllschlucker-Chöre*, V. 3 (WIEDEMANN 2003, p. 240). 


\section{c. Pappeln}

58. Ich hörte sagen (WIEDEMANN 2003, p. 63). - Landschaft (WIEDEMANN 2003, p. 51). Notturno (Wiedemann 2003, p. 393). - Unstetes Herz (Wiedemann 2003, p. 50); zur Deutung vgl. Werner, Textgräber [Anm. 29], p. 17-52.

\section{Sachkultur}

59. Mischkrug: In der ewigen Teufe, V. 8 (WIEDEMANN 2003, p. 223).

60. Pfeil: Der Pfeil der Artemis* (Wiedemann 2003, p. 17). - Sichtbar-Unsichtbar, V. 10 (WiedeMANN 2003, p. 498).

61. Siebenflöte: Aus den nahen*, V. 13 (WIEDEMANN 2003, p. 232).

62. Stierkopfgefäß: Entwurf einer Landschaft (WIEDEMANN 2003, p. 107-108). s. auch (Nr. 68).

\section{Skythen}

63. Sichtbar-unsichtbar, V. 2-3: „Ein / blauweißer Skythe, / zebragewandet, beritten“ (WIEDEMANN 2003, p. 498).

64. Und mit dem Buch aus Tarussa*, V. 35: „Skythisch / zusammengereimt“" (WiedeMAnN 2003, p. 164-166).

65. Filzschwäne: Schwanengefahr (Wiedemann 2003, 303). - Ungewaschen, unbemalt (WiedEMANN 2003, p. 315).

\section{Begriffe antiken Ursprungs}

66. Anabasis* (WiedEMANN 2003, p. 147-148). s. auch (Nr. 13).

67. Argumentum e silentio* (WIEDEMANN 2003, p. 86).

68. Brandmal (WIEDEMANN 2003, p. 43, vgl. p. 612).

69. Corona* (WIEDEMANN 2003, p. 39).

70. Das Gastmah* (WIEDEMANn 2003, p. 33). In der DA wird ein Bezug auf Platon vorgeschlagen. s. auch (Nr. 10).

\section{Begriffe aus der altertumswissenschaftlichen Literatur}

Halbzerfressener, V. 2: „Kragstein“ (WiEDEMANN 2003, p. 195, vgl. p. 736).

Unter der Flut, V. 3: ,gehöhten“ (WiEDEMANN 2003, p. 299, vgl. p. 822).

Wanderstaude, passim (WIEDEMANn 2003, p. 349); zur Deutung vgl. BollaCK 2006, p. 279299. 
